\title{
Über Steroid-Konjugate in Plasma
}

VI. Isolierung von Cortisol und Tetrahydrocortisol aus unpolaren, solvolysierbaren Konjugatfraktionen')

Von

Eberhard Kaiser und Georg W. Oertel

Aus der Endokrinologischen Abteilung, Institut für Hygiene und Mikrobiologie, Universität des Saarlandes, Homburg/Saar

(Direktor: Prof. Dr. Dr. W. Zimmermann)

(Der Schriftleitung zugegangen am 25. August 1962)

\begin{abstract}
Bei Aufarbcitung von Normal- und ACTH-Plasma sowie von vcrschiedenen, durch Chromatographie an DEAESephadex gewonnenen Serumproteinen konnten Porter-Silber-Chromogene aus ciner Fraktion unpolarer Steroidkonjugate isoliert werden. $\mathrm{Da}$ diese $\mathrm{C}_{21}$-Steroid-konjugate zusammen mit den als Sulfatidyl-17-ketosteroide angenommen $\mathrm{C}_{19}$-Steroid-konjugaten vorkamen und wie diese durch Solvolyse gespalten wurden, könntc auch ihnen die Struktur von Sulfatidyl-steroiden zugeschrieben werden. Signifikante Mengen an Porter-Silber-Chromogenen ließen sich in $\alpha$-Globulin-haltigen Proteinfraktionen nachweisen. Versuche zur Isolicrung einzelner $C_{21}$-Steroide aus der unpolaren Konjugatfraktion führten zum Nachweis von Cortisol und Tetrahydrocortisol.
\end{abstract}

Porter-Silber chromogens were isolated from a non-polar stcroid conjugate fraction, which was obtaincd from normal and ACTH plasma and various scrum proteins separated by chromatography on DEAE Sephadex. 'These $\mathrm{C}_{21}$-steroid conjugates were assigned the structure of sulphatidyl-steroids, because they occurred with $\mathrm{C}_{19}$-stcroid conjugates (assumed to be sulphatidyl-17-oxosteroids) and were also cleaved by solvolysis. Significant amounts of Porter-Silber chromogens could be demonstrated in protein fractions containing $\alpha$-globulin. Attempts to isolate individual $\mathrm{C}_{21}$-stcroids from the non-polar conjugate fraction resulted in the demonstration of cortisol and tetrahydrocortisol.

In vorausgegangenen Mitteilungen (1-4) berichteten wir über die Isolierung unpolarer 17-Ketosteroid-konjugate, vornehmlich aus $\alpha$-Globulin-haltigen Plasmafraktionen. $\mathrm{Da}$ diese Konjugate bei Chromatographie an DEAE-Zellulose zusammen mit anderen Lipoiden schon mittels Chloroform-Methanol-Wasser eluiert werden konnten und auch in Zusammensetzung, Beständigkeit und papierchromatographischem Verhalten weitgehend synthetischen Sulfatidyl-17-ketosteroiden glichen, wurde für die unpolaren Konjugate aus Plasma die Struktur letzterer Verbindungen angenommen. Für den Transport derartiger Verbindungen im Plasma sind offenbar Lipoproteine verantwortlich. Die Tatsache, daß über $80 \%$ der im Plasma enthaltenen 17Ketosteroid-konjugate ursprünglich in unpolarer Form vorlagen, ließ vermuten, daß möglicherweise sämtliche im Harn als Sulfate ausgeschiedenen Steroide im Plasma als Sulfatid ylverbindungen zirkulieren. Letztere scheinen dabei an spezifische Plasmaproteine, wie $\alpha_{1}$-Lipoproteine gebunden zu sein, was für andere Lipoide hinlänglich bekannt ist $(5,6$.$) 17-Ketosteroidsulfate, wie sie aus$ peripherem Plasma isoliert wurden $(7,8)$ stellen wahrscheinlich Hydrolyseprodukte von Sulfatidyl-17-ketosteroiden dar.

Nach den jüngsten Veröffentlichungen von PasQaLINI (9-11) besteht ein großer Teil der im Harn enthaltenen Konjugate von Corticosteroiden und deren Metaboliten

1) Vorliegende Arbeit wurde mit Unterstützung der Deutschen Forschungsgemeinschaft, Bad Godesberg, durchgeführt. aus Sulfaten. Die vorliegende Arbeit befaßt sich daher mit Versuchen zur Isolierung von unpolaren $C_{21}$ Steroidkonjugaten aus Plasmaextrakten oder entsprechenden Proteinfraktionen.

\section{Versuche}

\section{Fraktionierung von Plasma oder Serum}

Die Auftrennung von jeweils $100 \mathrm{ml}$ Plasma oder Serum, welches z. T. nach Verabreichung von 40 I. E. ACTH gewonnen wurde; erfolgte im wesentlichen nach früher beschriebenen Verfahren (3). ILediglich der $0,02 \mathrm{~m}$ Phosphatpuffer von $\mathrm{pH}_{\mathrm{H}}-6,7$ wurde durch einen solchen von $\mathrm{PH}-6,6$ ersetzt und die Chromatographie statt bei $15-17^{\circ} \mathrm{C}$ bei $3-4^{\circ} \mathrm{C}$ durchgeführt, um die Zersetzung labiler Proteine und Konjugate zu vermeiden.

\section{Aufarbeitung von Plasma oder Proteinfraktionen}

Die mittels Papierelektrophorese auf ihre Komponenten untersuchten und eingeengten Serumfraktionen (etwa $50 \mathrm{ml}$ ) wurden ebenso wie unbehandeltes Plasma dreimal mit je 1,5 Vol eiskaltem Methylenchlorid extrahiert, wodurch sich freie Corticosteroide weitgehend entfernen lassen. Es folgte die Extraktion der wäßrigen Phase nach Vorschrift von NYE u. a. (12) mit viermal je 4 Vol Chloroform-Methanol $(1: 1 \mathrm{v} / \mathrm{v})$. Die Trockenrückstände des Gesamtextraktes wurden sodann einer Säulenchromatographie an DEAE-Zellulose (Schleicher \& Schüll) unterworfen, indem man diese 
mittels dreimal je $2.5 \mathrm{ml}$ Chloroform-Methanol-Wasser $(1: 9: 2 \mathrm{v} / \mathrm{v})$ auf eine mit nämlichem Lösungsmittelgemisch zubereitete Säule (Durchmesser: $2 \mathrm{~cm}$ ) aus 5-15 g DEAE-Zellulose (je nach Menge des zu chromatographierenden Materials) überführte, mit etwas Chloroform-Methanol-Wasser (1:9:2 v/v) nachspülte und schließlich mit je $100 \mathrm{ml}$ folgender Lösungsmittel eluierte:

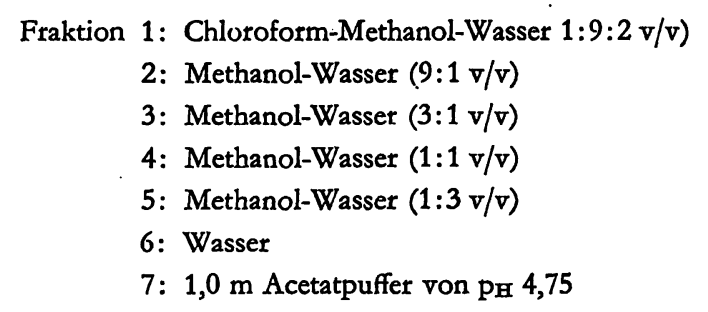

Die Vorbehandlung der DEAE-Zellulose geschah in üblicher Weise durch Waschen mit 0,1 n Salzsäure, Wasser, 0,1 n Natronlauge, Wasser und 90\% Methanol. Da nach Vorversuchen die Hauptmenge der im Plasma enthaltenen 17-Ketosteroid-konjugate und konjugierten -Porter-Silber-Chromogene in den Fraktionen 1 und 2 auftrat, während in Fraktion 7 lediglich Spuren der genannten Konjugate zu finden waren, wurden die beiden ersten Fraktionen zur Trockne eingedampft und der Rückstand einer Lösungsmittelverteilung zwischen Wasser und Benzol-Äthylazetat $(3: 1 \mathrm{v} / \mathrm{v})$ unterworfen. Für die Spaltung der in der wäßrigen Phase befindlichen Glucuronoside empfahl sich die enzymatische Hydrolyse mit $1000 \mathrm{E} \beta$-Glucuronidase/ml Lösung bei einem $p_{H}$ von 4,75 und einer Inkubationsdauer von 48 Stunden bei $37^{\circ} \mathrm{C}$. Zur weiteren Reinigung unpolarer Konjugate in der Benzol-Äthylazetat-Lösung wurde diese im Vakuum eingedampft und der Rückstand an Aluminiumoxyd (Woelm, Akt. Stufe 1, neutral) chromatographiert. Nach Auftragen des Rückstands mittels dreimal je $5 \mathrm{ml}$ Azeton-Äthanol (19:1 $\mathrm{v} / \mathrm{v})$ auf die mit dem gleichen Lösungsmittel hergestellte Säule (Durchmesser: $1 \mathrm{~cm}$ ) aus $10-20 \mathrm{~g}$ Aluminiumoxyd erfolgte die Elution mit je $50 \mathrm{ml}$ nachstehender Lösungsmittel:

$$
\begin{aligned}
& \text { Fraktion 1: Azeton-Äthanol (19:1 v/v) } \\
& \text { 2: Azeton-Äthanol }(4: 1 \mathrm{v} / \mathrm{v}) \\
& \text { 3: Azeton-Äthanol }(2: 1 \mathrm{v} / \mathrm{v}) \\
& \text { 4: Azeton-Äthanol }(1: 3 \mathrm{v} / \mathrm{v}) \\
& \text { 5: Azeton-Äthanol }(1: 9 \mathrm{v} / \mathrm{v})
\end{aligned}
$$

Früheren Versuchen zufolge befanden sich die unpolaren Konjugate in den beiden ersten Fraktionen, während in den Fraktionen 4 und 5 Steroid-sulfate auftraten. Fraktion 1 und 2 wurden daher vereinigt, zur Trockne eingedampft und der Rückstand einer Solvolyse in Äthylazetat unterworfen.

\section{Bestimmung und Identifizierung von Steroiden}

Die Fraktionen der freien, der durch $\beta$-Glucuronidase und der durch Solvolyse in Freiheit gesetzten Steroide wurden wie üblich durch Waschen mit Natronlauge und Wasser gereinigt, über Natriumsulfat getrocknet und unter Stickstoff zur Trockne eingedampft. Die Entfernung verunreinigender Lipoide erfolgte durch Ausfrieren in $70 \%$ Methanol bei $-15^{\circ} \mathrm{C}$. Anschließend wurde der Trockenrückstand der methanolischen Lösung im Lösungsmittelsystem Propylenglykol/Methylcyclohexan (13) chromatographiert. Die anhand ihres $\mathbf{R}_{\mathrm{T}}$-Wertes ermittelten Zonen von Dehydroepiandrosteron, Aetiocholanolon und Androsteron wurden eluiert und die Rückstände der betreffenden Eluate mittels der ZrmmermanN-Reaktion quantitativ analysiert. Unter Verwendung entsprechender Standardverbindungen wurden sodann die in der Nähe des Auftragungsorts zurückgebliebenen Corticosteroide in den Lösungsmittelsystemen Propylenglykol/Toluol (72-96 Stunden) (14) und Äthylazetat-Toluol/Methanol-Wasser $(1: 9: 5: 5 \mathrm{v} / \mathrm{v})$ (15) papierchromatographisch aufgetrennt, die Tetrazolium-positiven, vermutlich Cortisol und Tetrahydro-Cortisol enthaltenden Abschnitte jeweils eluiert und aliquote Teile der Eluate dann mittels der Porter-Silber-Reaktion (16) auf $17-\mathrm{Hy}-$ droxy-20,21-ketole untersucht. Es erschien weiterhin angezeigt, das UV-Absorptionsspektrum beider Eluate aufzunehmen und mit aliquoten Teilen eine Tetrazoliumblau-Reaktion $(17,18)$ durchzuführen. Die Hauptmenge der in dem ersten Eluat befindlichen, als Cortisol angesehenen Verbindung I wurde mit $0,1 \mathrm{ml}$ Essigsäureanhydrid in 0,1 ml Pyridin während $18 \mathrm{Stun}$ den bei Zimmertemperatur azetyliert und das Reaktionsprodukt neben Cortisol-Azetat-Standard im Lösungsmittelsystem Formamid/Benzol bzw. Propylenglykol/Toluol chromatographiert. Die Cortisol-Azetat entsprechende Verbindung wurde eluiert und durch Chromatographie an Aluminiumoxyd (Woelm; Akt. Stufe 1, neutral) endgültig gereinigt. Hierzu überführte man das Azetat mittels Benzol auf die mit Benzol zubereitete Säule von $1 \mathrm{~g}$ Aluminiumoxyd, spülte mit $10 \mathrm{ml} 0,2 \%$ Methanol in Benzol nach und eluierte sodann mit $20 \mathrm{ml}$ 10\% Methanol in Benzol. Letztere Fraktion enthielt Cortisol-Azetat, welches einer Messung von UV- und Schwefelsäure-Absorptionsspektrum zugeführt wurde (19).

Für die Charakterisierung der Porter-Silber-positiven Substanz II mit der Wanderungsgeschwindigkeit von Tetrahydrocortisol wählten wir die Oxydation mittels Natriumwismutat (20), die durch Auflösen des Rückstandes in $1 \mathrm{ml} 50 \%$ Essigsäure, einstündige Behandlung mit 12,5 mg Natriumwismutat, Zugabe vọ $1 \mathrm{ml}$ $6 \%$ Natriummetabisulfit und übliche Extraktion des Oxydationsproduktes effolgte. Nach anschließender Papierchromatographie des entstandenen 17-Ketosteroids in Formamid/Benzol und Propylenglykol/Toluol wurde dieses schließlich durch Chromatographie an $0,5 \mathrm{~g}$ Aluminiumoxyd gereinigt, wobei Benzol zur Herstellung der Säule und zum Aufbringen des Oxydationsproduktes, 7,5\% Methanol in Benzol aber zur Elution des 17-Ketosteroids Verwendung fand. MikroZimmermann-Reaktion und Schwefelsäure-Absorptionsspektrum vervollständigten die Identifizierung. 
Tab. 1

Eigenschaften der aus Plasma isolierten Verbindung I

\begin{tabular}{|c|c|c|}
\hline Eigenschaften & Verbindung I & Cortisol-Standarc \\
\hline $\begin{array}{lr}\text { Wanderungsgeschwindigkeit in System } & 1 \\
\text { Wanderungsgeschwindigkeit in System } & 2 \\
\text { Wanderungsgeschwindigkeit, nach Azetylierung, in System } 1 \\
\text {.Wanderungsgeschwindigkeit, nach Azetylierung, in System } 3 \\
\text { Porter-Silber-Reaktion } \\
\text { Tetrazoliumblau-Reaktion } \\
\text { UV-Absorptionsmaximum in Methanol } \\
\text { Absorptionsmaxima des Azetats in } \mathrm{H}_{2} \mathrm{SO}_{4} \\
\text { (Nebenmaxima) }\end{array}$ & $\begin{array}{c}R_{E}=0,42 \\
R_{E^{\prime}}=0,33 \\
R_{W^{\prime}}=2,76 \\
R_{F^{\prime}}=0,19 \\
\text { positiv } \\
\text { positiv } \\
241 \mathrm{~m} \mu \\
241,284 \mathrm{~m} \mu \\
(346,472 \mathrm{~m} \mu)\end{array}$ & $\begin{array}{c}0,44 \\
0,34 \\
2,85 \\
0,21 \\
\text { positiv } \\
\text { positiv } \\
242 \mathrm{~m} \mu \\
242,285 \mathrm{~m} / \mu \\
(348,476 \mathrm{~m} \mu)\end{array}$ \\
\hline
\end{tabular}

Sys:em 1 = Propylenglykol/Toluol

$2=$ Athylazetat-Toluol/Methanol-Wasser (1:9:5:5 v/v)

$3=$. Formamid/Benzol

$R_{E}=$ Wanderungsgeschwindigkeit bezogen auf Cortison

Tab. 2

Eigenschaften der aus Plasma isolierten Verbindung II

\begin{tabular}{|c|c|c|c|}
\hline Eigenschaften & & Verbindung II & Tetrabydrocortisol-Standard \\
\hline $\begin{array}{l}\text { Wanderungsgeschwindigkeit in System } \\
\text { Wanderungsgeschwindigkeit in System } \\
\text { Wanderungsgeschwindigkeit, nach Oxydation, in System } \\
\text { Wanderungsgeschwindigkeit in System } \\
\text { Porter-Silber-Reaktion } \\
\text { Tetrazoliumblau-Reaktion } \\
\text { UV-Absorptionsmaximum in Methanol } \\
\text { Absorptionsmaximum des Oxydationsproduktes in } \mathrm{H}_{2} \mathrm{SO}_{4} \\
\text { (Nebenmaximum) }\end{array}$ & $\begin{array}{l}1 \\
2 \\
3 \\
1\end{array}$ & $\begin{array}{l}R_{E}=0,10 \\
R_{F}=0,19 \\
R_{T}=0,31 \\
R_{F}=0,28 \\
\quad \text { positiv } \\
\text { positiv } \\
\text { keines } \\
316 \mathrm{~m} \mu \\
(258 \mathrm{~m} \mu)\end{array}$ & $\begin{array}{l}0,11 \\
0,18 \\
0,31 \\
0,27 \\
\text { positiv } \\
\text { positiv } \\
\text { keines } \\
318 \mathrm{~m} \mu \\
(255 \mathrm{~m} \mu)\end{array}$ \\
\hline
\end{tabular}

System $1=$ Propylenglykol/Toluol $2=$ Athylazetat-Toluol/Methanol-W/asser $(1: 9: 5: 5 \mathrm{v} / \mathrm{v})$ $3=$ Formamid/Benzol

\section{Ergebnisse}

Nach der Entfernung der freien Steroide durch Extraktion mit Methylenchlorid, welches - je nach aufgearbeiteter Plasmaprobe $-, 12,5-17,3 \mu \mathrm{g}$ PorterSilber-Chromogene pro $100 \mathrm{ml}$ Plasma enthielt, fand sich die Hauptmenge der 17-Ketosteroid-konjugate, wie auch ein großer Teil der Corticosteroid-konjugate bei Chromatographie an DEAE-Zellulose oder Aluminiumoxyd in den Lipoid-haltigen Fraktionen 1 und 2 (Abbildung 1). Setzten sich die von DEAE-Zellulose eluierten Konjugate in den Fraktionen 1 und 2 noch aus Glucuronosiden und unpolaren, solvolysierbaren Konjugaten zusammen, so führte die Lösungsmittelverteilung $z$ wischen Wasser und Benzol-Äthylazetat (3:1 $v / v)$ zur Abtrennung einer 11,2-17,3 $\mu \mathrm{g}$ Porter-SilberChromogene umfassenden Fraktion, welche nach Bebrütung mit $\beta$-Glucuronidase aus der wäßrigen Phase extrahiert werden konnte. Die gleichzeitig anfallende unpolare Konjugatfraktion in der organischen Phase erbrachte im Anschluß an Solvolyse insgesamt 7,9$14,2 \mu \mathrm{g}$ an Porter-Silber-Chromogenen pro $100 \mathrm{ml}$ Plasma. Während bei der ersten Chromatographie an DEAE-Zellulose $\quad 0,4-3,9 \mu \mathrm{g}$ Porter-Silber-Chromogene in der mit Puffer herausgelösten Fraktion 7 enthalten waren und gleichfalls durch Solvolyse in Freiheit
$R_{E}=$ Wanderungsgescbwindigkeit bezogen auf Cortison

$R_{T}=$ Wanderungsgeschwindigkeit bezogen auf Testosteron
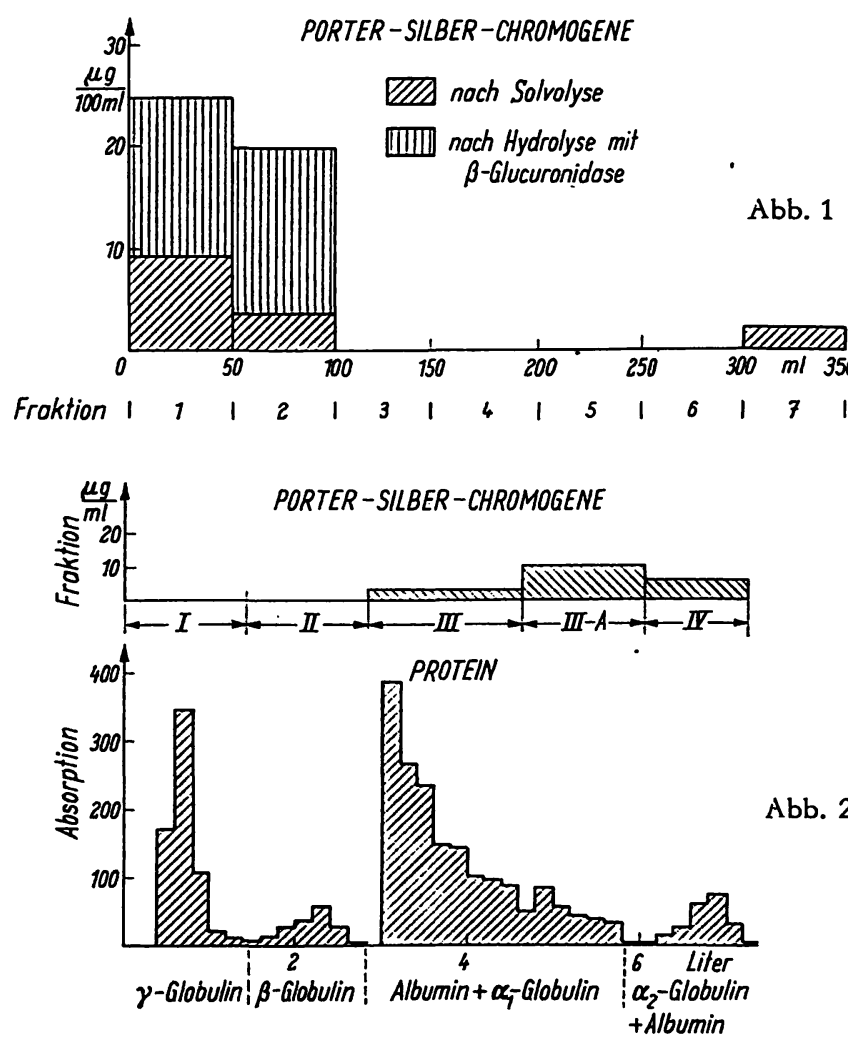

(Absorption bei $280 \mathrm{~m} \mu$ ). 
gesetzt wurden, ließen sich bei nachfolgender Säulenchromatographie der unpolaren Konjugatfraktion weitere $0,6-1,1 \mu \mathrm{g}$ Porter-Silber-Chromogene in den Eluaten 4 und 5 der Aluminiumoxydsäule nachweisen. Versuche, die unpolaren Konjugate einer bestimmten Proteinkomponente des Plasmas zuzuordnen, zeitigten die in Abbildung 2 zusammengefaßten Ergebnisse. Ebenso wie 17-Ketosteroid-konjugate enthielten die aus Albumin, $\alpha_{1}$ - und $\alpha_{2}$-Globulin bestehenden Fraktionen III-A und IV auch Porter-Silber-Chromogene in ursprünglich unpolarer Bindung. Tabelle 1 und 2 zeigen die Eigenschaften der isolierten Verbindungen I und II im Vergleich mit denen der jeweiligen Standardsubstanzen. Angesichts der Úbereinstimmung in den angewandten Farbreaktionen, der Beweglichkeit der freien Verbindung und eines Derivats in verschiedenen Lösungsmittelsystemen der Papierchromatographie sowie im UV- und Schwefelsäure-absorptionsspektrum erscheint die Identifizierung von Verbindung I und II als Cortisol und Tetrahydrocortisol hinlänglich gesichert.

\section{Diskussion}

Nachdem Pasqualini $(9,10)$ im menschlichen Harn größere Mengen an Corticosteroid-sulfaten gefunden hatte, war ein Vorkommen derartiger $\mathrm{C}_{21}$-Steroid-konjugate im Plasma gleichfalls zu erwarten, zumal die Clearance von Steroid-sulfaten offenbar hinter der von Steroid-glucuronosiden zurückbleibt, wie Untersuchungen von Bongiovanni u. Eberlein (21) und Kellie u. SMITH (22) im Falle der 17-Ketosteroide eindeutig erkennen ließen. $\mathrm{Da}$ nach unserer Auffassung die im
Harn ausgeschiedenen 17-Ketosteroid-sulfate nur $\mathrm{Hy}-$ drolyseprodukte der im Plasma zirkulierenden Sulfatidyl-17-ketosteroide darstellen, sollten die vermuteten Porter-Silber-Chromogene wie diese in lipoidhaltigem Material auftreten. Tatsächlich waren PorterSilber-Chromogene in den ersten Eluaten der DEAEZellulosesäule wie auch der Aluminiumoxyd-säule enthalten. Versuche, die Porter-Silber-Chromogene zu identifizieren, führten zum Nachweis von Cortisol und Tetrahydrocortisol, während Corticosteron nicht einwandfrei nachgewiesen werden konnte. Desweiteren blieben zusätzliche Tetrazoliumblau-positive Zonen des ersten Chromatogramms von polaren Corticosteroiden unberücksichtigt, so daß mit dem Vorkommen weiterer solvolysierbarer $\mathrm{C}_{21}$-Steroid-konjugate zu rechnen ist. Die benutzten Identifizierungsverfahren beschränkten sich auf die Wanderungsgeschwindigkeit der freien Verbindungen und geeigneter Derivate in verschiedenen papierchromatographischen Systemen, Porter-Silberund Tetrazoliumblau-Reaktion sowie UV- und Schwefelsäure-Absorptionsspektrum. (Die Messung des Infrarot-Absorptionsspektrums fehlt infolge der niedrigen Konzentration beider Steroide in den isolierten unpolaren Fraktionen.) Es ist interessant, daß offenbar nicht nur das freie Cortisol an ein $\alpha$-Globulin, nämlich das sogenannte "Transcortin“, gebunden ist (23-25), sondern auch das erstmals isolierte, solvolysierbare Cortisol-konjugat, wie die Fraktionierung von Serum an DEAE-Sephadex ergab. Ob es sich hier um das gleiche Protein handelt, kann zur Zeit nicht entschieden werden. Versuche, die mit Sulfatidyl-steroiden assoziierten $\alpha$-Globuline in reiner Form zu gewinnen und mit Transcortin zu vergleichen, sind im Gange.

\section{Literatur}

1. Oertel, G. W., Biochem. Z. 334, 431 (1961). - 2. Oertel, G. W. und E. Karser, Clin. Chim. Acta 7, 463 (1962). - 3. Oertel, G. W. und E. Karser, Biochem. Z. 336, 10 (1962). - 4. Oertel, G. W. und E. Karser, Biochem. Z. 336, 154 (1962). - 5. GuRd, F. R. N., in "Lipide Chemistry“, Hanahan, D. J. Edit., John Wiley u. Sons, 1960, New York, S. 208-260. - 6. ONCLEX, J. L., F. R. N. Gurd and M.'Melvin, J. Am. Chem. Soc. 72, 458 (1950).7. Baulieu, E. E., J. clin. Endocrinol. Metab. 20, 900 (1960). 8. Baulieu, E. E., Experientia 17, 110 (1961). - 9. Pasqualini, J. R., Compt. rend. Séances Acad. Sci. 250, 1929 (1960). - 10. Pasqualini, J. R., Compt. rend. Seances Acad. Sci. 246, 2945 (1958). - 11. Pasqualini, J. R., Thesis, Faculté de Médicine de Paris, 1962. - 12. NyE, W. R., C. Waterhouse and G. V. MarINETTI, J. clin. Invest. 40, 1194 (1961). - 13. SAVARD, K., J. biol.
Chemistry 202, 457 (1953). - 14. Zaffaroni, A., Rec. Progr Hormone Res. 8, 51 (1953). - 15. Bush, I. E., Biochem. J. 50, 370 (1952). - 16. Porter, C. C. and R. H. Silber, J. biol. Chemistry 185, 201 (1950). - 17. MADER, W. J. and R. R. BuCK, Analytic. Chem. 24, 666 (1952). - 18. Weichselbaum, T. E. and H. W. Margraf, J. clin. Endocrinol. Metab. 15, 970 (1955). - 19. Zaffaroni, A., J. Amer. Chem. Soc. 72, 3828 (1950). - 20. NorymberskI, J. K., Nature [London] 170, 1074 (1952). - 21. Bongrovanni, A. M. and W. R. Eberdein, J. clin. Endocrinol. Metab. 17, 238 (1957). - 22. Kellie, A. E. and E. R. SMIth, Biochem. J. 66, 490 (1957: - 23. Daughaday, W. H., J. clin. Invest. 35, 1528 (1956). - 24. Daughaday, W. H., J. clin. Invest. 37, 519 (1958). - 25. Slaunwhite, W. R. and A. A. Sandberg, J. clin. Invest. 38, 384 (1959).
Dozent Dr. G. W. Oertel Universitäts-Institut für Hygiene und Mikrobiologie 665 Homburg / Saar 